\title{
The 'tipping point' applied
}

\author{
Stephen B Hanauer
}

The epiphany came while I was lecturing on the Positioning of biologic agents to treat inflammatory bowel disease at an annual Crohn's \& Colitis Foundation of America conference. As I wrote in my November 2005 editorial, it can readily be debated that anti-tumor necrosis factor agents are the most efficacious treatment for IBD and can reduce side effects (compared with corticosteroids), hospitalizations and surgeries. Only around 50\% of patients ever require steroids, however, and with data from Olmsted County demonstrating that the prognosis for both Crohn's disease (Gastroenterology 114: 1161-1168) and ulcerative colitis patients after initiating steroids is grim-including a nearly $30 \%$ need for surgery within 1 year-the revelation struck me that the need for steroid therapy is a 'tipping point' within the course of IBD.

In his book The Tipping Point, which was first published in 2000, Malcolm Gladwell describes the characteristics of epidemics as "...contagiousness; the fact that little causes can have big effects; and, that change happens not gradually but at one dramatic moment..." with the latter being the tipping point. It is clear to me that this applies to the course of IBD; the moment of dramatic change provides a clinically logical discerning feature that can be used to determine when to initiate biologic therapy.

Now, as I contemplate other disease states and peruse the medical literature, the concept of a tipping point frequently jumps to mind. We have used various terminologies to describe the deflection point of a disease course, determining it using symptoms, signs, laboratory values or other diagnostic strategies.

In this journal we have reviewed the metabolic syndrome and the impact of mesenteric fat.
... .tipping

points provide

opportunities

in medicine to

potentially alter

the course of

a disease or

epidemic

SB Hanauer is Editorin-Chief of Nature Clinical Practice Gastroenterology \& Hepatology.

\section{Competing interests}

The author declared he has no competing interests.

www.nature.com/clinicalpractice doi:10.1038/ncpgasthep0411
One example of a tipping point related to the metabolic syndrome can be found in an article on The metabolic syndrome as a predictor of nonalcoholic fatty liver disease by Hamaguchi and colleagues (Ann Intern Med 143: 722-728). These investigators followed over 4,000 healthy Japanese men and women with a mean BMI of $22.6 \mathrm{~kg} / \mathrm{m}^{2}$ for approximately 1 year. At baseline, 18\% had evidence (by liver ultrasound) of nonalcoholic fatty liver disease (NAFLD). During the follow-up $10 \%$ of the initial cohort developed ultrasound changes of NAFLD and $16 \%$ of the initial cohort had regression of NAFLD (i.e. $89 \%$ of those who had evidence of NAFLD at baseline). Not only were men and women with the metabolic syndrome at baseline more likely to develop NAFLD (odds ratio 4.0, 95\% Cl 2.63-6.08 and odds ratio 11.20, 95\% Cl 4.85-25.87, respectively), but the development of fatty liver was associated with an increase in BMI of $1 \mathrm{~kg} / \mathrm{m}^{2}$ (approximately $2-3 \mathrm{~kg}$ ) and, similarly, regression of fatty liver was associated with a small mean weight loss (2.3-2.5 kg). In other words, small changes in weight can be the tipping point for the development of NAFLD and other consequences of the metabolic syndrome.

We can appreciate the concept of tipping points in many other diseases, irrespective of epidemics. These are the deflection points at which time a disease course changes direction, amplitude or acceleration. In many cases it can be the 'straw that broke the camel's back'. In any case, we should be cognizant of these "...dramatic moment(s) when...everything can change all at once..." because such tipping points provide opportunities in medicine to potentially alter the course of a disease or epidemic. 